\title{
ON THE OSTWALD RIPENING OF THIN LIQUID FILMS*
}

\author{
SHIBIN DAI
}

\begin{abstract}
Dewetting instabilities cause a thin liquid film coating a solid substrate to rupture and finally form complex patterns, which are quasiequilibrium parabolic droplets connected by an ultra thin residual film. During the Ostwald ripening process, droplets exchange mass through the residual thin film without touching each other. Bigger ones grow while smaller ones shrink and disappear. As a result the total number of droplets $N(t)$ decreases while the average size increases. For the physically realistic case when the underlying substrate is two dimensional, it is predicted that the average volume of droplets $\mathcal{V}$ follows a temporal power-logarithmic law: $\mathcal{V}^{4 / 3} \ln \mathcal{V} \sim c t$. We propose a mean field model for the Ostwald ripening of $2 \mathrm{D}$ thin films and define a structural time scale $t_{s}$, which is heuristically similar to $t$. In this mean field model we rigorously prove that $\mathcal{V}$ can not grow faster than the power-logarithmic law in $t_{s}$ in the average sense, as long as the droplets are well separated.
\end{abstract}

Key words. Thin film equation, coarsening, Ostwald ripening, mean field models.

AMS subject classifications. 35B40, 76A20, 35K25, 35K55, 35Q80.

\section{Introduction}

Due to the dewetting instabilities, a thin liquid film coating a solid substrate goes through complicated morphological changes and ultimately forms complex nonlinear patterns in the late stage. The patterns are essentially quasi-static fluid droplets connected by an ultra thin residual film. Droplets may exchange mass through a diffusion field in the ultra thin film. Smaller ones shrink and collapse while bigger ones grow. This mechanism is called Ostwald ripening, as it is similar to what happens in phase transitions (see, e.g., [22]). Meanwhile, droplets may move around and collide to form bigger ones. These two mechanisms cause coarsening phenomena to occur where we observe the decrease of the total number of droplets and an increase in the average droplet size and the average distance between droplets.

For the simplified case when the underlying substrate is one-dimensional, the coarsening dynamics of the thin film was studied by Glasner and Witelski [10, 12] using asymptotic analysis methods. Heuristic arguments and numerical simulations suggest that under both mechanisms the number of droplets $N(t)$ decreases following a temporal power law $N(t) \sim c t^{-2 / 5}$. As a consequence, the average distance between drops grows as a temporal power law $t^{2 / 5}$ and the average height and width of drops grow as $t^{1 / 5}$. The size distribution of droplets was studied in [13] by considering a mean field model when the ripening mechanism dominates. Their numerical simulations indicate that the power law for $N(t)$ holds in the average sense when self-similarity of the size distribution is not attained. In [2], we studied the mean field model in [13] and rigorously proved that in the average sense $N(t)$ can not decrease faster than the $t^{-2 / 5}$ power law.

When the underlying substrate is two dimensional, which is the physically realistic case, the situation becomes much more complicated. It is shown in [9] and [11] that the shapes of liquid droplets are paraboloids. The contact angles are fixed, independent of the size of droplets. The contact angles are the angles between the surface of the

*Received: February 3, 2010; accepted (in revised version): May 20, 2010. Communicated by Andrea Bertozzi.

${ }^{\dagger}$ Department of Mathematical Sciences, Worcester Polytechnic Institute, Worcester, MA 01609, USA (sdai@wpi.edu). 
droplets and the uniform ultra thin residual film. Because of this feature, the droplets are totally determined by their circular bases.

In [9] Glasner used asymptotic matching methods to study the roles of ripening and collision and heuristically concluded that the ripening mechanism indicated that the characteristic volume $\mathcal{V}$ of droplets increases as a temporal power law $t^{3 / 4}$, with a logarithmic correction coefficient

$$
\mathcal{V}^{4 / 3} \ln \mathcal{V} \sim c t .
$$

We call (1.1) a power-logarithmic law. In [11], Glasner et al. studied the same problem using energy arguments and heuristically obtained the same result. Other results in $[9,11]$ include that the collision mechanism can be as important as the ripening and can induce similar growth rates of characteristic sizes.

No rigorous result is known so far for the power-logarithmic law (1.1). The goal of this paper has two parts. First we propose a mean field model for the two dimensional thin film when the ripening mechanism dominates. In our mean field model, the power-logarithmic law becomes a built-in feature. The second part of our goal is to give some rigorous analysis for (1.1) in the mean field setting. We obtain an estimate on the coarsening rate that is consistent with the power-logarithmic law (1.1) in a "structural time scale" $t_{s}$. We call $t_{s}$ a "structural timescale" because it captures some statistical information of the distribution. It will be discussed in detail later.

One specific advantage of a mean field model is that it enables us to obtain statistical information about the distribution of droplets such as the number and average size of droplets. It is generally conjectured that the size distribution of liquid droplets takes a self-similar form. Numerical simulations also somewhat agree with such a conjecture, but rigorous justification is lacking. A major challenge to rigorous analysis is to find an easy way to retrieve such statistical information from the nonlinear thin film equation itself. In a mean field model, the size distribution of droplets obeys a transport equation. We can study the existence (or nonexistence) and properties of self-similar solutions for such an equation.

We describe our mean field model as follows (a formal derivation will be given in Section 2). Suppose at time $t$, there are $N(t)$ droplets on a square substrate $\Omega=[0, \mathcal{L}]^{2}$ and let $\left\{R_{i}, i=1, \cdots, N(t)\right\}$ be the radii of their bases. Since we do not consider the migration and collision of droplets, the spatial locations of droplets are not essential as long as the droplets are well-separated (note however that the migration can be significant in thin films). Our mean field model says that the evolution of radii must satisfy the following evolution law:

$$
\begin{gathered}
\dot{R}_{i}=-\frac{1}{R_{i}^{2}} \frac{1}{\ln \phi(t)^{-1}}\left(\frac{1}{R_{i}}-P_{*}(t)\right) \quad \text { for all } i=1, \cdots, N, \\
\phi(t)=\frac{\sum_{i=1}^{N} R_{i}^{2}}{\mathcal{L}^{2}} \text { and } P_{*}(t)=\frac{\sum_{i=1}^{N} R_{i}^{-1}}{N} .
\end{gathered}
$$

Here $\phi$ is the rescaled fraction of the substrate that is covered by the bases of the droplets and $P_{*}$ is a mean field determined by the conservation of total volume of droplets. That is, it is a result of $\sum_{i=1}^{N} R_{i}^{2} \dot{R}_{i}=0 . P_{*}$ then determines a critical radius $\mathcal{R}_{*}=P_{*}^{-1}$ which is the harmonic mean of all radii $\left\{R_{i}\right\}$. Note that $\phi$ and $P_{*}$ are spatial constants but vary with time. From (1.2), since $\phi$ is always smaller than 1 , droplets with radii bigger than $\mathcal{R}_{*}$ grow and those with radii smaller than $R_{*}$ shrink and finally disappear. 
Singularities occur when some radii approach zero. After the singular moments, we remove the droplets that disappeared from the system and continue.

Now we consider the evolution of the distribution of radii. Let $f(t, R)$ be the density function of the distribution of radii at time $t$. Given the evolution law for radii (1.2), the transport equation for $f$ is immediately written as

$$
\frac{\partial}{\partial t} f(t, R)+\frac{\partial}{\partial R}\left(-\frac{1}{R^{2}} \frac{1}{\ln \phi^{-1}}\left(\frac{1}{R}-P_{*}\right) f(t, R)\right)=0 .
$$

Here

$$
\phi=\frac{\int_{0}^{\infty} R^{2} f(t, R) d R}{\mathcal{L}^{2}}, \quad P_{*}=\frac{\int_{0}^{\infty} R^{-1} f(t, R) d R}{\int_{0}^{\infty} f(t, R) d R}
$$

The minimum requirement on $f(t, R)$ is $\int_{0}^{\infty} R^{2} f(t, R) d R<\mathcal{L}^{2}$ since the droplets can not occupy all the substrate. In addition, the conservation of total volume of droplets translates into $\int_{0}^{\infty} R^{3} f(t, R) d R=$ const.

The evolution law (1.2) has one spectacular property; it makes it easy to capture the power-logarithmic law (1.1) for the coarsening rate because of the built-in factor $\ln \phi^{-1}$.

The relation between $\phi$ and the characteristic volume $\mathcal{V}$ of droplets is pretty simple. Take $\mathcal{V}$ to be the average volume

$$
\mathcal{V}_{\text {avg }}=\frac{\mathcal{V}_{\text {total }}}{N}
$$

Since the droplets are paraboloids with the same fixed contact angels, the characteristic radius of the droplet bases should be $\tilde{\mathcal{R}}=a \mathcal{V}_{\text {avg }}^{1 / 3}$ where $a$ is a constant determined by the fixed contact angle. Without loss of generality, after a simple rescaling we can just take $a=1$. By the definition (1.3) of $\phi$,

$$
\phi=\frac{\sum_{i=1}^{N} R_{i}^{2}}{\mathcal{L}^{2}} \sim \frac{N \tilde{\mathcal{R}}^{2}}{\mathcal{L}^{2}} \sim \frac{\mathcal{V}_{\text {total }} \mathcal{V}_{\text {avg }}^{2 / 3}}{\mathcal{V}_{\text {avg }} \mathcal{L}^{2}} \sim \frac{\mathcal{V}_{\text {total }}}{\mathcal{L}^{2}} \mathcal{V}_{\text {avg }}^{-1 / 3} \sim h_{\text {avg }} \mathcal{V}_{\text {avg }}^{-1 / 3}
$$

Here $h_{\text {avg }}:=\mathcal{V}_{\text {total }} / \mathcal{L}^{2}$ is the average height of the thin film, which is a constant determined by the initial configuration. So

$$
h_{\text {avg }} \phi^{-1} \sim \mathcal{V}_{\text {avg }}^{1 / 3}
$$

According to (1.1), the relation between $\phi^{-1}$ and time $t$ should be

$$
c t \sim \mathcal{V}_{\text {avg }}^{4 / 3} \ln \mathcal{V}_{\text {avg }} \sim h_{\text {avg }}^{4} \phi^{-4} \ln \left(h_{\text {avg }}^{3} \phi^{-3}\right) \sim h_{\text {avg }}^{4} \phi^{-4} \ln \left(\phi^{-3}\right)
$$

provided that $h_{\text {avg }}$ is of magnitude $O(1)$, which is always reasonable by an appropriate nondimensionalization of the system. So

$$
\phi\left(\ln \phi^{-1}\right)^{-1 / 4} \sim c_{1} h_{\text {avg }} t^{-1 / 4},
$$

where $c_{1}=(c / 3)^{-1 / 4}$.

Our main result about the coarsening rate of the mean field model (1.2)-(1.3) is a one-sided time averaged estimate that is consistent with (1.10). Namely we prove that $\phi\left(\ln \phi^{-1}\right)^{-1 / 4}$ can not decrease faster than the $t_{s}^{-1 / 4}$ power law in the "structural 
time scale" $t_{s}$, in the average sense. Consequently we prove that the average volume $\mathcal{V}_{a v g}$ of droplets can not increase faster than the power-logarithmic law (1.1), i.e.,

$$
\mathcal{V}_{a v g}^{4 / 3} \ln \mathcal{V}_{a v g} \leqslant c t_{s}
$$

in the average sense. The structural time scale $t_{s}$ captures some of the statistical information of the size distribution of droplets. Its properties need to be explored but it is always true that $t_{s} \geqslant t$ and heuristically $t_{s} \sim t$.

Our analysis employs a method introduced by Kohn and Otto in [14], where they consider the coarsening rates for two Cahn-Hilliard equations, one with constant mobility and the other with degenerate mobility. Their results are universal timeaveraged power law lower bounds for decay of a normalized free energy. These power law bounds are consistent with the coarsening rates of characteristic length scales. They are called universal because they apply to every solution, are independent of any system parameters, and independent of statistical assumptions.

This method consists of three key ingredients:

(i) an interpolation inequality between the normalized energy and an auxiliary dual quantity;

(ii) a dissipation inequality that connects the rates of change of the free energy and of the dual quantity;

(iii) an ODE argument based on the two inequalities gives the desired timeaveraged power law lower bound on the energy.

The Kohn-Otto framework has been applied successfully to many different cases $[1,2,3,4,5,6,7,8,15,16,21]$. One common feature of these applications is that the systems under consideration dissipate a normalized energy in a rate as a temporal power law and all ultimate estimates are bounds on the normalized energy. The auxiliary dual quantities are constructed in various ways. Some are constructed by taking advantage of the gradient flow structure of the dynamics [1, 6, 7, 8, 15, 16, 21], some by the simple geometric morphologies $[2,3,5]$, and some by the combination of both [4].

We want to point out that the results in [21] are about a nonlinear thin film PDE with such a mobility that droplet collisions are absent according to [9]. Their results capture the leading order power law of $\mathcal{V} \sim t^{3 / 4}$ but do not capture the logarithmic factor $\ln \mathcal{V}$. Hence in some sense our results are improvements of those in [21].

Indeed it is the logarithmic factor that distinguish our results from those in [21].

In our model, the system dissipates the surface energy, which is proportional to $\phi$. However, what we want to prove is a power-logarithmic law (1.10), not a power law for $\phi$ vs. $t$. If we define $\Phi:=\phi\left(\ln \phi^{-1}\right)^{-1 / 4}$, then we have a power law relation between $\Phi$ and $t$. It is not clear if $\Phi$ can be interpreted as energy in some sense. To apply the Kohn-Otto framework, we need to find a quantity dual to $\Phi$.

First we define a quantity that is dual to $\phi$. Because of the parabolic shapes of droplets, we apply the ideas in [5] and define an auxiliary quantity

$$
\sigma(t):=\frac{\mathcal{L}^{2} \sum_{i=1}^{N} R_{i}^{4}}{\mathcal{V}_{\text {total }}^{2}}
$$

where $\mathcal{V}_{\text {total }}=\sum_{i=1}^{N} R_{i}^{3}$ is the conserved total volume of droplets. $\sigma(t)$ is "dual" to 
$\phi(t)$ in the sense that the interpolation inequality holds

$$
\phi(t) \sigma(t)=\frac{\left(\sum_{i=1}^{N} R_{i}^{2}\right)\left(\sum_{i=1}^{N} R_{i}^{4}\right)}{\left(\sum_{i=1}^{N} R_{i}^{3}\right)^{2}} \geqslant 1 .
$$

Note that the definition of $\sigma$ is purely out of mathematical necessity, even though it can be interpreted as the normalized $4^{\text {th }}$ moment of the distribution of droplet radii.

To construct a quantity that is dual to $\Phi$, we define $S:=\sigma(\ln \sigma)^{1 / 4}$. It is immediate that the interpolation inequality holds

$$
\Phi(t) S(t) \geqslant 1
$$

As for the dissipation relation, due to technical reasons we need the "structural" time scale

$$
t_{s}:=\int_{0}^{t}\left(\frac{\ln \sigma(s)}{\ln \phi(s)^{-1}}\right)^{1 / 2} d s
$$

We call $t_{s}$ a "structural" time scale because it captures some statistical information of the distribution of droplets - for example, if all radii are identical, then $t_{s}=t$. In general since $\sigma \geqslant \phi^{-1}>1, t_{s}$ is an increasing function of $t$ and $t_{s} \geqslant t$.

Rewriting $\Phi$ and $S$ in the structural time scale $t_{s}$, we can have a dissipation inequality (details will be given in Section 3). Finally, by applying an ODE argument we have the following estimate.

TheOREM 1.1. Assume that the initial droplet configuration of the thin liquid film satisfies $\phi<e^{-1}$. Then for any $2<p<4$ there exist positive constants $C_{1}$ and $C_{2}$ depending on $p$ and independent of any system parameter such that for any solutions $\left\{R_{i}\right\}$ of the mean field model (1.2)-(1.3), if we rewrite $\phi(t)$ in $t_{s}$, i.e., if $\tilde{\phi}\left(t_{s}\right)=\phi(t)$, then

$$
\int_{0}^{T_{s}}\left(\tilde{\phi}\left(t_{s}\right)\left(\ln \tilde{\phi}^{-1}\left(t_{s}\right)\right)^{-1 / 4}\right)^{p} d t_{s} \geqslant C_{2} \int_{0}^{T_{s}}\left(h_{\text {avg }} t_{s}^{-1 / 4}\right)^{p} d t_{s}
$$

provided $T_{s}>C_{1} h_{\text {avg }}^{4} \sigma(0)^{4} \ln \sigma(0)$.

An estimate on the growth rate of the average volume of droplets can be obtained as a consequence of Theorem 1.1.

THEOREM 1.2. In addition to the assumption in Theorem 1.1, assume further that the initial configuration satisfies $\mathcal{V}_{\text {avg }} \geqslant h_{\text {avg }}^{3}$. For any $2<p<4$, let $C_{1}, C_{2}$ be the same constants as in Theorem 1.1. Then for any solutions $\left\{R_{i}\right\}$ of the mean field model (1.2)-(1.3), if we rewrite the average volume of droplets $\mathcal{V}_{\text {avg }}$ in $t_{s}$, i.e., if $\tilde{\mathcal{V}}\left(t_{s}\right)=\mathcal{V}_{\text {avg }}(t)$, then

$$
\int_{0}^{T_{s}}\left\{\tilde{\mathcal{V}}\left(t_{s}\right)^{4 / 3} \ln \left(\frac{\tilde{\mathcal{V}}\left(t_{s}\right)}{h_{\text {avg }}^{3}}\right)\right\}^{-p / 4} d t_{s} \geqslant 3^{-p / 4} C_{2} \int_{0}^{T_{s}} t_{s}^{-p / 4} d t_{s}
$$

provided $T_{s}>C_{1} h_{\text {avg }}^{4} \sigma(0)^{4} \ln \sigma(0)$.

REMARK 1.3. Theorem 1.2 says that if the system is nondimensionalized in a way such that $h_{\text {avg }}$ is of order $O(1)$, then in the average sense $\tilde{\mathcal{V}}\left(t_{s}\right)^{4 / 3} \ln \tilde{\mathcal{V}}\left(t_{s}\right) \leqslant c t_{s}$, which 
is an upper bound of the coarsening rate that is consistent with the power-logarithmic law (1.1). Specifically the constant $c$ is independent of any system parameter.

REMARK 1.4. The bounds in Theorems 1.1 and 1.2 are not only one-sided, but also in the time average sense. These limitations can not be removed without considering detailed statistical information of the dynamics. We can not obtain universal two sided bounds because of the existence of systems that coarsen slower than the powerlogarithmic law. For example, there are unstable equilibrium states.

As for pointwise bounds, it is shown in [13] that there exist "staircasing" transient dynamics, which are very different from self similar dynamics, in the Ostwald ripening of thin films for some initial droplet distributions. This annihilates the possibility of a one-sided pointwise power law bound. Nevertheless, numerical simulations show that, even in these "staircasing" transient dynamics, the time-averaged coarsening rates always obey the same power law as in self similar dynamics. This in part justifies that a time averaged bound is the best we can expect without considering statistical information of the dynamics.

REMARK 1.5. We discuss the assumptions in Theorems 1.1 and 1.2. The assumption $\phi<e^{-1}$ is obviously reasonable since droplets will cover only a small part of the substrate. Nevertheless we can get a little more information when combined with the assumption $\mathcal{V}_{\text {avg }} \geqslant h_{\text {avg }}^{3}$ in Theorem 1.2. Since

$$
\mathcal{V}_{\text {total }}=h_{\text {avg }} \mathcal{L}^{2}, \quad \mathcal{V}_{\text {avg }}=\frac{\mathcal{V}_{\text {total }}}{N}, \quad \text { and } \quad \frac{\mathcal{V}_{a v g}}{h_{\text {avg }}^{3}}=\frac{\mathcal{L}^{2}}{N h_{\text {avg }}^{2}},
$$

if we define

$$
\gamma:=\frac{\mathcal{V}_{\text {avg }}}{h_{\text {avg }}^{3}}=\frac{\mathcal{L}^{2}}{N h_{\text {avg }}^{2}}
$$

then

$$
N=\frac{1}{\gamma} \frac{\mathcal{L}^{2}}{h_{a v g}^{2}}
$$

The assumption $\mathcal{V}_{\text {avg }} \geqslant h_{\text {avg }}^{3}$ in Theorem 1.2 requires $\gamma \geqslant 1$. In addition, as will be proved in Section 3,

$$
\phi=\frac{\sum_{i=1}^{N} R_{i}^{2}}{\mathcal{L}^{2}} \leqslant h_{a v g} \mathcal{V}_{\text {avg }}^{-1 / 3}=\left(\frac{N h_{\text {avg }}^{2}}{\mathcal{L}^{2}}\right)^{1 / 3}=\frac{1}{\gamma^{1 / 3}} .
$$

The assumption in Theorem 1.1 can be achieved by choosing $\gamma=e^{3}$. Combined with (1.19), the regime we study is when the initial configuration satisfies

$$
N \approx \frac{1}{e^{3}} \frac{\mathcal{L}^{2}}{h_{a v g}^{2}} .
$$

Since the starting point of our mean field model is when the system aspect ratio is large, i.e., when $\mathcal{L}$ is several orders of magnitude bigger than $h_{\text {avg }}$, systems under our consideration can be very complex with a big number of droplets.

The rest of the paper is structured as follows. In Section 2 we give a heuristic derivation of our mean field model (1.2)-(1.3). In Section 3 we will prove Theorems 1.1 and 1.2. In Section 4 we will discuss the relation between the structural time scale $t_{s}$ and the self-similarity of the distribution of droplet radii. Finally in Section 5 we discuss possible generalizations to our mean field model and results. 


\section{Formal derivation of the mean field model}

In this section, we derive the mean field model for physically realistic thin liquid films with 2D underlying substrate. We only consider the case when the ripening mechanism dominates the dynamics and the migration and collisions of droplets can be ignored.

Mathematically, the thin film dynamics are described by the lubrication theory [20]. Denote by $h=h(x, t)$ the height of the thin film. The evolution of $h$ satisfies the following equation

$$
\partial_{t} h+\nabla \cdot\left(h^{q} \nabla\left(\Delta h-U^{\prime}(h)\right)\right)=0,
$$

where $U(h)$ is an intermolecular potential that includes both attractive and repulsive effects and the balance produces a single minimum $h_{\min }$ for $h$, which corresponds to the globally stable ultra thin film. The only mathematically necessary properties of the potential $U$ are:

$$
\begin{aligned}
& U(h) \text { has a unique minimum } h_{\text {min }}, \\
& U^{\prime}(h) \text { has a unique maximum, } p_{\text {max }} \text {, at the maximum droplet height } h_{\text {max }}, \\
& U^{\prime}(h)=o\left(h^{-1}\right) \text { and } U(h) \rightarrow 0 \text {, as } h \rightarrow \infty, \\
& \lim _{h \rightarrow 0} U(h)=\infty
\end{aligned}
$$

The mobility $h^{q}$ depends on boundary conditions when deriving the thin film equation from the Stokes equation. For example, the standard nonslip boundary condition gives a mobility corresponding to $q=3$.

When the underlying substrate is $2 \mathrm{D}$, the shapes of liquid droplets are paraboloids with fixed contact angles that are determined solely by the intermolecular potential $U(h)$. Because of this feature, the droplets are totally determined by their circular bases.

In [9] and [11], it is pointed out that the motion of the droplet bases is determined by the mobility in the nonlinear thin film Equation (2.1). When the power $0<q<3$, the influence of the mobility of the droplets are minor compared to the ripening process. Hence we can ignore the collision of droplets and just concentrate on the ripening process.

In this case, our mean field model to the coarsening is a transport equation for the distribution of the radii of bases. When the distribution is discrete, it becomes a system of ODEs. This system has singularities when some droplets shrink and disappear.

Suppose that at time $t$ there are $N(t)$ droplets. Let $\left\{R_{i}, i=1, \cdots, N\right\}$ be the radii of their bases and $\left\{X_{i}, i=1, \cdots, N\right\}$ be the centers of the bases. Denote by $\Gamma_{i}:=$ $\left\{x \in \mathbb{R}^{2}:\left|x-X_{i}\right|=R_{i}\right\}$ the boundary of the $i^{\text {th }}$ base. The evolution of the droplets is determined by the variation of the hydradynamic pressures. Since the liquid droplets are quasistationary, it is pointed out in [9] that after a trivial rescaling, the variation of pressure $P$ in the thin residual film satisfies

$$
\begin{aligned}
-\Delta P(x) & =0 \quad \text { if }\left|x-X_{i}\right|>R_{i} \text { for all } i \\
P & =\frac{1}{R_{i}} \quad \text { on } \Gamma_{i}, \\
\dot{R}_{i} & =\frac{1}{R_{i}^{2}} \int_{\Gamma_{i}}[\nabla P \cdot n] d s \quad \text { on } \Gamma_{i} .
\end{aligned}
$$


Here $[\nabla P \cdot n]$ is the jump of the normal gradient of $P$ across the boundary. Boundary condition (2.4) guarantees that the total volume of droplets is preserved during the ripening process. Note that because of the parabolic shapes, volumes of droplets are proportional to the cube of their radii of the bases.

We will derive a mean field model from Equations (2.2)-(2.4). We consider the case when the distance between droplets are much bigger than the size of droplets. Then, at a given time, near the droplets the pressure $P$ should be similar to the fundamental solution to the 2D Laplacian operator. Away from droplets, $P$ will approach a mean field $P_{*}$. This indicates that we require a "cutoff" of the fundamental solution. Assume $d$ is the cutoff distance, that is, $d$ is a number bigger than droplet sizes but much smaller than the distance between droplets so that $P$ becomes the mean field $P_{*}$ at points $x$ when $\left|x-X_{i}\right| \geqslant d$ for all $i$. Then $P$ should have the following form near the $i^{\text {th }}$ droplet,

$$
P(x)=c_{i} \ln \frac{\left|x-X_{i}\right|}{d}+P_{*} \quad \text { for }\left|x-X_{i}\right| \leqslant d .
$$

REMARK 2.1. The mean field $P_{*}$ is the average effect of neighbor droplets and it varies in a length scale $\mathcal{L}_{s}$ called the screening length, which measures the distance droplets' influence can reach (about screening length see, e.g., [17, 18]). In a thin film, according to [17], if $l$ is the average distance between droplets and $R$ is the typical radius of droplets, then

$$
\mathcal{L}_{s}^{2} \approx l^{2} \ln \frac{l}{R} .
$$

Taking $\delta:=\mathcal{L} / \mathcal{L}_{s}$ to be the relative size of the system compared to the screening length and $\epsilon:=l / \mathcal{L}$ as a dimensionless parameter for the average distance between droplets, we have

$$
\delta^{-2} \approx \epsilon^{2} \ln \frac{l}{R}, \quad R \approx l \exp \left(-\delta^{-2} \epsilon^{-2}\right) .
$$

The fraction $\phi$ then satisfies

$$
\phi \approx \frac{R^{2}}{l^{2}} \approx \exp \left(-2 \delta^{-2} \epsilon^{-2}\right) .
$$

When $\mathcal{L}_{s} \gg \mathcal{L}, P_{*}$ is a spatial constant that varies with time. In this paper, we will only consider this case. Our mean field model corresponds to the dilute limit when $\phi \rightarrow 0$ in a rate given above and both $\delta \rightarrow 0$ and $\epsilon \rightarrow 0$.

The boundary condition (2.3) requires that

$$
\frac{1}{R_{i}}=c_{i} \ln \frac{R_{i}}{d}+P_{*},
$$

and hence

$$
c_{i}=\frac{\left(\frac{1}{R_{i}}-P_{*}\right)}{\ln \frac{R_{i}}{d}} .
$$

From the boundary condition (2.4) we obtain an evolution law for $R_{i}$ :

$$
\dot{R}_{i}=\frac{2 \pi}{R_{i}^{2}} c_{i}=\frac{2 \pi}{R_{i}^{2}} \frac{1}{\ln \frac{R_{i}}{d}}\left(\frac{1}{R_{i}}-P_{*}\right) .
$$


Note that the cutoff distance $d$ is artificial and the coefficient $\left(\ln \frac{R_{i}}{d}\right)^{-1}$ is still hard to work with. Heuristically we may choose $d$ to be the average distance between the droplets. Since the thin film covers a 2 dimensional $\mathcal{L} \times \mathcal{L}$ square domain, we take $N d^{2}=\mathcal{L}^{2}$, or $d=\mathcal{L} / \sqrt{N}$.

In the coefficient $\left(\ln \frac{R_{i}}{d}\right)^{-1}$, without changing the leading order behavior of the model, we may replace $R_{i}$ by the characteristic radius $\mathcal{R}$ of all droplets. $\mathcal{R}$ is characteristic in the sense that $N \mathcal{R}^{2}$ equals the total area covered by all the bases of droplets. That is,

$$
N \mathcal{R}^{2}=\sum_{i=1}^{N} R_{i}^{2}, \quad \text { or } \quad \mathcal{R}=\left(\frac{\sum_{i=1}^{N} R_{i}^{2}}{N}\right)^{1 / 2} .
$$

We define $\mathcal{R}$ in such a way because we only consider the two dimensional feature of the underlying substrate, hence the two dimensional feature of the pressure field $P$.

Now the coefficient $\left(\ln \frac{R_{i}}{d}\right)^{-1}$ is replaced by

$$
\left(\ln \frac{\mathcal{R}}{d}\right)^{-1}=\left(\ln \frac{\sqrt{N} \mathcal{R}}{\sqrt{N} d}\right)^{-1}=\left(\frac{1}{2} \ln \frac{\sum_{i=1}^{N} R_{i}^{2}}{\mathcal{L}^{2}}\right)^{-1} .
$$

The coefficient (2.10) is changing with time and it has some profound connection with the coarsening dynamics.

As is in Section 1, define

$$
\phi(t):=\frac{\sum_{i=1}^{N} R_{i}^{2}}{\mathcal{L}^{2}}
$$

Then (2.10) is related to $\phi$,

$$
\left(\ln \frac{\mathcal{R}}{d}\right)^{-1}=\left(\frac{1}{2} \ln \phi\right)^{-1}=-\left(\frac{1}{2} \ln \phi^{-1}\right)^{-1} .
$$

Replacing the coefficient $\left(\ln \frac{R_{i}}{d}\right)^{-1}$ in (2.9) by (2.12) and rescaling time, we obtain our evolution law for radii, which is a coupled ODE system.

(a.) Evolution law for radii. For all $i=1, \cdots, N$, we have

$$
\dot{R}_{i}=-\frac{1}{R_{i}^{2}} \frac{1}{\ln \phi^{-1}}\left(\frac{1}{R_{i}}-P_{*}\right) .
$$

The mean field $P_{*}$ is determined by the conservation of the total volume of droplets. That is, $\sum_{i=1}^{N} R_{i}^{2} \dot{R}_{i}=0$ implies that

$$
P_{*}=\frac{\sum_{i=1}^{N} R_{i}^{-1}}{N} .
$$

\section{(b.) The transport equation for the distribution of radii}

Now we consider an arbitrary distribution $f(t, R)$ of radii at time $t$. Given the evolution law for radii (2.13), the transport equation for $f$ is

$$
\frac{\partial}{\partial t} f(t, R)+\frac{\partial}{\partial R}\left(-\frac{1}{R^{2}} \frac{1}{\ln \phi^{-1}}\left(\frac{1}{R}-P_{*}\right) f(t, R)\right)=0 .
$$


Here

$$
\phi=\frac{\int_{0}^{\infty} R^{2} f(t, R) d R}{\mathcal{L}^{2}}, \quad P_{*}=\frac{\int_{0}^{\infty} R^{-1} f(t, R) d R}{\int_{0}^{\infty} f(t, R) d R} .
$$

Remark 2.2. Equations (2.2)-(2.4) are similar to the 2D Mullins-Sekerka model for phase transitions when one phase consists of circular particles. Equation (2.3) is the Gibbs-Thomson condition on the boundary. The difference lies in Equation (2.4); in the 2D Mullins-Sekerka model for phase transitions, it is the total area enclosed by the boundaries $\left\{\Gamma_{i}\right\}$ that is preserved. Hence the 2D Mullins-Sekerka model has a boundary condition

$$
\dot{R}_{i}=\frac{1}{R_{i}} \int_{\Gamma_{i}}[\nabla P \cdot n] d s \quad \text { on } \Gamma_{i}
$$

REMARK 2.3. The formal derivation of our mean field model is similar to that in [17], where Niethammer and Otto formally derived and rigorously justified a mean field model for domain coarsening of $2 \mathrm{D}$ phase transitions. Note that in 2D phase transitions, since $\sum_{i=1}^{N} R_{i}^{2}$ is conserved, the above coefficient $(2.10)$ is a constant.

\section{Estimates on the coarsening rate}

In this section we prove the lower bound on the dissipation rate of $\phi(t)(1.15)$ and the upper bound on the growth rate of the average volume $\mathcal{V}_{\text {avg }}(1.16)$.

First we discuss the relation between $\phi(t)$ and the free energy of the system. Since the morphology of the thin film is parabolic droplets connected by a uniform ultra thin residual film, the free energy is just the surface energy of droplets. The surface energy is proportional to the surface area of all droplets and hence proportional to $\sum_{i=1}^{N} R_{i}^{2}$. So $\phi(t)=\sum_{i=1}^{N} R_{i}^{2} / \mathcal{L}^{2}$ is the rescaled free energy of the system and it should be decreasing.

In fact since

$$
\dot{R}_{i}=-\frac{1}{R_{i}^{2}} \frac{1}{\ln \left(\phi(t)^{-1}\right)}\left(\frac{1}{R_{i}}-P_{*}\right)
$$

the dissipation rate of $\phi(t)$ is

$$
\dot{\phi}(t)=\frac{2}{\mathcal{L}^{2}} \sum_{i=1}^{N} R_{i} \dot{R}_{i}=-\frac{2}{\mathcal{L}^{2}} \sum_{i=1}^{N} \frac{1}{R_{i}} \frac{1}{\ln \phi^{-1}}\left(\frac{1}{R_{i}}-P_{*}\right) \quad \text { by (3.1). }
$$

Since the total volume $\mathcal{V}_{\text {total }}=\sum_{i=1}^{N} R_{i}^{3}$ is conserved, we have

$$
0=\sum_{i=1}^{N} R_{i}^{2} \dot{R}_{i}=-\sum_{i=1}^{N} \frac{1}{\ln \left(\phi(t)^{-1}\right)}\left(\frac{1}{R_{i}}-P_{*}\right) .
$$

By $(3.2),(3.3)$, and the fact that $P_{*}$ is a spatial constant, we obtain

$$
\begin{aligned}
\dot{\phi}(t) & =-\frac{2}{\mathcal{L}^{2}}\left(\sum_{i=1}^{N} \frac{1}{R_{i}} \frac{1}{\ln \phi^{-1}}\left(\frac{1}{R_{i}}-P_{*}\right)-P_{*} \sum_{i=1}^{N} \frac{1}{\ln \phi^{-1}}\left(\frac{1}{R_{i}}-P_{*}\right)\right) \\
& =-\frac{2}{\mathcal{L}^{2}} \sum_{i=1}^{N} \frac{1}{\ln \phi^{-1}}\left(\frac{1}{R_{i}}-P_{*}\right)^{2} \leqslant 0 \quad(\text { since } \phi<1) .
\end{aligned}
$$


Next we define an auxiliary quantity

$$
\sigma(t):=\frac{\mathcal{L}^{2} \sum_{i=1}^{N} R_{i}^{4}}{\mathcal{V}_{\text {total }}^{2}}
$$

$\sigma(t)$ is "dual" to $\phi(t)$ in the sense that the following interpolation inequality holds.

LEMMA 3.1. (Interpolation inequality)

$$
\phi(t) \sigma(t) \geqslant 1 \text {. }
$$

Proof. This is an immediate consequence of the Cauchy-Schwarz inequality.

$$
\phi(t) \sigma(t)=\frac{\left(\sum_{i=1}^{N} R_{i}^{2}\right)\left(\sum_{i=1}^{N} R_{i}^{4}\right)}{\left(\sum_{i=1}^{N} R_{i}^{3}\right)^{2}} \geqslant 1 .
$$

In addition, the following inequality holds between the dissipation rates of $\phi(t)$ and $\sigma(t)$.

Lemma 3.2. (Dissipation inequality)

$$
(\dot{\sigma})^{2} \leqslant 8 h_{\text {avg }}^{-4} \frac{1}{\ln \phi^{-1}} \phi(-\dot{\phi}),
$$

where $h_{\text {avg }}=\mathcal{V}_{\text {total }} / \mathcal{L}^{2}$.

Proof. By (3.1) and (3.4), $\dot{\phi}$ can be rewritten as

$$
\dot{\phi}=-\frac{2}{\mathcal{L}^{2}} \ln \phi^{-1} \sum_{i=1}^{N} R_{i}^{4} \dot{R}_{i}{ }^{2} .
$$

Direct calculation and the Cauchy-Schwarz inequality show that

$$
\begin{aligned}
|\dot{\sigma}|=\frac{4 \mathcal{L}^{2}}{\mathcal{V}_{\text {total }}^{2}}\left|\sum_{i=1}^{N} R_{i}^{3} \dot{R}_{i}\right| & \leqslant \frac{4 \mathcal{L}^{2}}{\mathcal{V}_{\text {total }}^{2}}\left(\sum_{i=1}^{N} R_{i}^{2}\right)^{1 / 2}\left(\sum_{i=1}^{N} R_{i}^{4}\left(\dot{R}_{i}\right)^{2}\right)^{1 / 2} \\
& =\frac{4 \mathcal{L}^{2}}{\mathcal{V}_{\text {total }}^{2}}\left(\mathcal{L}^{2} \phi\right)^{1 / 2}\left(-\frac{\mathcal{L}^{2}}{2 \ln \phi^{-1}} \dot{\phi}\right)^{1 / 2}
\end{aligned}
$$

So

$$
(\dot{\sigma})^{2} \leqslant \frac{8 \mathcal{L}^{8}}{\mathcal{V}_{\text {total }}^{4}} \frac{1}{\ln \phi^{-1}} \phi(-\dot{\phi})=8 h_{\text {avg }}^{-4} \frac{1}{\ln \phi^{-1}} \phi(-\dot{\phi}) .
$$

Inequalities (3.6) and (3.8) are the starting points of our proof for Theorem 1.1. However since Theorem 1.1 is about $\phi\left(\ln \phi^{-1}\right)^{-1 / 4}$, we define

$$
\Phi:=\phi\left(\ln \phi^{-1}\right)^{-1 / 4},
$$


and correspondingly

$$
S:=\sigma(\ln \sigma)^{1 / 4} .
$$

We will apply Lemmas 3.1 and 3.2 to obtain the following interpolation and dissipation inequalities involving $\Phi, S, \dot{\Phi}$, and $\dot{S}$.

Lemma 3.3. (Interpolation inequality for $S$ and $\Phi$ )

$$
S(t) \Phi(t) \geqslant 1
$$

Proof. By (3.6), $\sigma \geqslant \phi^{-1}>1$. So

$$
S(t) \Phi(t)=\sigma(t) \phi(t)\left(\frac{\ln \sigma}{\ln \phi^{-1}}\right)^{1 / 4} \geqslant 1
$$

Lemma 3.4. (Dissipation inequality for $\dot{S}$ and $\dot{\Phi})$ Assume that $\phi(0)<e^{-1}$. Then

$$
(\dot{S})^{2} \leqslant 16 h_{\text {avg }}^{-4}\left(\frac{\ln \sigma}{\ln \phi^{-1}}\right)^{1 / 2} \Phi(-\dot{\Phi}) .
$$

Proof. Since $\phi(t)$ is a decreasing function of $t, \phi(0)<e^{-1}$ implies that $\phi(t)<e^{-1}$ and $\ln \phi(t)^{-1}>1$ for all $t \geqslant 0$. Direct calculation shows that

$$
\begin{aligned}
\dot{\Phi} & =\dot{\phi}\left(\ln \phi^{-1}\right)^{-1 / 4}\left(1+\frac{1}{4}\left(\ln \phi^{-1}\right)^{-1}\right), \\
\Phi(-\dot{\Phi}) & =\phi(-\dot{\phi})\left(\ln \phi^{-1}\right)^{-1 / 2}\left(1+\frac{1}{4}\left(\ln \phi^{-1}\right)^{-1}\right), \\
\dot{S} & =\dot{\sigma}(\ln \sigma)^{1 / 4}\left(1+\frac{1}{4}(\ln \sigma)^{-1}\right) .
\end{aligned}
$$

Consequently

$$
\begin{aligned}
(\dot{S})^{2} & =(\dot{\sigma})^{2}(\ln \sigma)^{1 / 2}\left(1+\frac{1}{4}(\ln \sigma)^{-1}\right)^{2} \\
& \leqslant 8 h_{\text {avg }}^{-4} \frac{(\ln \sigma)^{1 / 2}}{\ln \left(\phi^{-1}\right)} \phi(-\dot{\phi})\left(1+\frac{1}{4}(\ln \sigma)^{-1}\right)^{2} \quad \text { by (3.8). }
\end{aligned}
$$

Since $\sigma \phi \geqslant 1$, we have $\ln \sigma \geqslant \ln \phi^{-1}>1$, and $\left(1+\frac{1}{4}(\ln \sigma)^{-1}\right)^{2}<2\left(1+\frac{1}{4}\left(\ln \phi^{-1}\right)^{-1}\right)$. So

$$
\begin{aligned}
(\dot{S})^{2} & \leqslant 16 h_{\text {avg }}^{-4} \frac{(\ln \sigma)^{1 / 2}}{\ln \left(\phi^{-1}\right)} \phi(-\dot{\phi})\left(1+\frac{1}{4}\left(\ln \phi^{-1}\right)^{-1}\right) \\
& =16 h_{\text {avg }}^{-4}\left(\frac{\ln \sigma}{\ln \phi^{-1}}\right)^{1 / 2} \Phi(-\dot{\Phi}) \quad \text { by }(3.18) .
\end{aligned}
$$

$\square$ Now we are ready to prove Theorem 1.1. Define a structural time scale

$$
t_{s}:=\int_{0}^{t}\left(\frac{\ln \sigma(s)}{\ln \phi^{-1}(s)}\right)^{1 / 2} d s
$$


Then

$$
\frac{d t_{s}}{d t}=\left(\frac{\ln \sigma(t)}{\ln \phi^{-1}(t)}\right)^{1 / 2} \geqslant 1
$$

and hence $t_{s}$ is a strictly increasing function of $t$ and $t_{s} \geqslant t$. Then $S(t)$ and $\Phi(t)$ can be transformed into functions of $t_{s}$ as

$$
\tilde{S}\left(t_{s}\right):=S(t) \quad \text { and } \quad \tilde{\Phi}\left(t_{s}\right):=\Phi(t),
$$

and the dissipation inequality (3.16) becomes

$$
\left(\frac{d}{d t_{s}} \tilde{S}\right)^{2} \leqslant 16 h_{\text {avg }}^{-4} \tilde{\Phi}\left(-\frac{d}{d t_{s}} \tilde{\Phi}\right)
$$

Furthermore, if we define $\tau=16 h_{\text {avg }}^{-4} t_{s}$ and rewrite $\tilde{S}\left(t_{s}\right), \tilde{\Phi}\left(t_{s}\right)$ in $\tau$ as

$$
\hat{S}(\tau):=\tilde{S}\left(t_{s}\right), \quad \hat{\Phi}(\tau):=\tilde{\Phi}\left(t_{s}\right),
$$

then the dissipation inequality (3.24) becomes

$$
\left(\frac{d}{d \tau} \hat{S}\right)^{2} \leqslant \hat{\Phi}\left(-\frac{d}{d \tau} \hat{\Phi}\right)
$$

Apparently the interpolation inequality (3.14) can be directly translated into

$$
\hat{S}(\tau) \hat{\Phi}(\tau) \geqslant 1 .
$$

Now we can apply the following ODE argument.

Lemma 3.5. ([14]) For any $2<p<4$, there exist positive constants $\hat{C}_{1}, \hat{C}_{2}$ depending solely on $p$ such that if two continuous quantities $E(t)$ and $L(t)$ satisfy

$$
E(t) L(t) \geqslant 1, \quad \text { and } \quad\left(\frac{d L}{d t}\right)^{2} \leqslant E\left(-\frac{d E}{d t}\right)
$$

then we have

$$
\int_{0}^{T} E(t)^{p} d t \geqslant \hat{C}_{2} \int_{0}^{T}\left(t^{-1 / 4}\right)^{p} d t \quad \text { provided } T \geqslant \hat{C}_{1} L(0)^{4}
$$

Taking $L(t)$ as $\hat{S}(\tau)$ and $E(t)$ as $\hat{\Phi}(\tau)$, we obtain the following theorem.

TheOREM 3.6. Assume that $\phi(0)<e^{-1}$. For any $2<p<4$, there exist positive constants $\hat{C}_{1}, \hat{C}_{2}$ depending on $p$ but not on any system parameter such that for any solutions $\left\{R_{i}\right\}$ of the mean field model (3.1), we have

$$
\int_{0}^{\hat{\tau}} \hat{\Phi}(\tau)^{p} d \tau \geqslant \hat{C}_{2} \int_{0}^{\hat{\tau}}\left(\tau^{-1 / 4}\right)^{p} d \tau \quad \text { provided } \hat{\tau} \geqslant \hat{C}_{1} \hat{S}(0)^{4}
$$

Changing the variable back into $t_{s},(3.30)$ becomes

$$
\int_{0}^{T_{s}} \tilde{\Phi}\left(t_{s}\right)^{p} d t_{s} \geqslant C_{2} \int_{0}^{T_{s}}\left(h_{a v g} t_{s}^{-1 / 4}\right)^{p} d t_{s} \quad \text { provided } T_{s} \geqslant C_{1} h_{\text {avg }}^{4} \tilde{S}(0)^{4}
$$


where $C_{1}=16^{-1} \hat{C}_{1}$ and $C_{2}=2^{-p} \hat{C}_{2}$. Inequality (3.31) is exactly Theorem 1.1.

Before proving Theorem 1.2, we consider the relations between $\phi, \mathcal{V}_{\text {avg }}, \mathcal{R}_{\text {avg }}$, and $\mathcal{R}_{*}$. Recall that the average volume $\mathcal{V}_{\text {avg }}$, the average radius $\mathcal{R}_{\text {avg }}$ and the critical radius $\mathcal{R}_{*}$ are defined as

$$
\mathcal{V}_{\text {avg }}=\frac{\mathcal{V}_{\text {total }}}{N}=\frac{\sum_{i=1}^{N} R_{i}^{3}}{N}, \quad \mathcal{R}_{\text {avg }}=\frac{\sum_{i=1}^{N} R_{i}}{N}, \quad \text { and } \quad \mathcal{R}_{*}=\frac{N}{\sum_{i=1}^{N} R_{i}^{-1}}
$$

Lemma 3.7. For any solution $\left\{R_{i}: i=1, \cdots, N\right\}$ of the mean field model (3.1), $h_{\text {avg }} \phi^{-1}(t)$ is an upper bound for the average radius $\mathcal{R}_{\text {avg }}$, the critical radius $\mathcal{R}_{*}$, and the cube root of the average volume of of droplets. In fact, these quantities are ordered as follows.

$$
\mathcal{R}_{*} \leqslant \mathcal{R}_{\text {avg }} \leqslant \mathcal{V}_{\text {avg }}^{1 / 3} \leqslant h_{\text {avg }} \phi^{-1}
$$

Proof. Inequalities in (3.33) are consequences of Hölder's inequality (or the Cauchy-Schwarz inequality in some cases). Note that $\mathcal{V}_{\text {total }}=\sum_{i=1}^{N} R_{i}^{3}$ and $\mathcal{L}^{2}=h_{\text {avg }}^{-1} \mathcal{V}_{\text {total }}$.

(1.) The Cauchy-Schwarz inequality shows that

$$
N^{2} \leqslant\left(\sum_{i=1}^{N} R_{i}\right)\left(\sum_{i=1}^{N} R_{i}^{-1}\right)
$$

Hence

$$
\mathcal{R}_{*}=\frac{N}{\sum_{i=1}^{N} R_{i}^{-1}} \leqslant \frac{\sum_{i=1}^{N} R_{i}}{N}=\mathcal{R}_{\text {avg }} .
$$

(2.) By Hölder's inequality,

$$
\sum_{i=1}^{N} R_{i} \leqslant N^{2 / 3}\left(\sum_{i=1}^{N} R_{i}^{3}\right)^{1 / 3}
$$

So

$$
\mathcal{R}_{a v g}=\frac{\sum_{i=1}^{N} R_{i}}{N} \leqslant\left(\frac{\sum_{i=1}^{N} R_{i}^{3}}{N}\right)^{1 / 3}=\mathcal{V}_{a v g}^{1 / 3}
$$

(3.) Again by Hölder's inequality

$$
\sum_{i=1}^{N} R_{i}^{2} \leqslant N^{1 / 3}\left(\sum_{i=1}^{N} R_{i}^{3}\right)^{2 / 3}=\left(\frac{N}{\mathcal{V}_{\text {total }}}\right)^{1 / 3} h_{\text {avg }} \mathcal{L}^{2} .
$$

So

$$
\phi=\frac{\sum_{i=1}^{N} R_{i}^{2}}{\mathcal{L}^{2}} \leqslant h_{\text {avg }}\left(\frac{N}{\mathcal{V}_{\text {total }}}\right)^{1 / 3}=h_{\text {avg }} \mathcal{V}_{\text {avg }}^{-1 / 3}
$$


or equivalently

$$
\mathcal{V}_{\text {avg }}^{1 / 3} \leqslant h_{\text {avg }} \phi^{-1}
$$

Proof of Theorem 1.2. In Theorem 1.1, the estimate (1.15) is a lower bound for $\tilde{\Phi}\left(t_{s}\right)=\tilde{\phi}\left(t_{s}\right)\left(\ln \tilde{\phi}\left(t_{s}\right)^{-1}\right)^{-1 / 4}$. What we need to do is to translate it into an estimate for $\mathcal{V}_{\text {avg }}$ using (3.33). Define

$$
w(x)=x\left(\ln x^{-1}\right)^{-1 / 4} .
$$

Then $\Phi=w(\phi)$. Since

$$
w^{\prime}(x)=\left(\ln x^{-1}\right)^{-1 / 4}\left(1+\frac{1}{4}\left(\ln x^{-1}\right)^{-1}\right)>0 \quad \text { for all } 0<x<1,
$$

$w(x)$ is an increasing function on $(0,1)$. By (3.33), if $h_{\text {avg }} \mathcal{V}_{\text {avg }}^{-1 / 3}<1$, then

$$
\phi \leqslant h_{a v g} \mathcal{V}_{a v g}^{-1 / 3}<1
$$

and we have $w(\phi) \leqslant w\left(h_{\text {avg }} \mathcal{V}_{\text {avg }}^{-1 / 3}\right)$, i.e.,

$$
\Phi \leqslant h_{\text {avg }} \mathcal{V}_{\text {avg }}^{-1 / 3}\left(\ln \left(h_{\text {avg }}^{-1} \mathcal{V}_{\text {avg }}^{1 / 3}\right)\right)^{-1 / 4}=h_{\text {avg }} 3^{1 / 4}\left\{\mathcal{V}_{\text {avg }}^{4 / 3} \ln \left(\frac{\mathcal{V}_{\text {avg }}}{h_{\text {avg }}^{3}}\right)\right\}^{-1 / 4} .
$$

Rewrite $\mathcal{V}_{\text {avg }}(t)$ in terms of the structural time scale $t_{s}$,

$$
\tilde{\mathcal{V}}\left(t_{s}\right):=\mathcal{V}_{a v g}(t)
$$

Equation (3.39) is

$$
h_{\text {avg }}^{-1} 3^{-1 / 4} \tilde{\Phi}\left(t_{s}\right) \leqslant\left\{\tilde{\mathcal{V}}\left(t_{s}\right)^{4 / 3} \ln \left(\frac{\tilde{\mathcal{V}}\left(t_{s}\right)}{h_{\text {avg }}^{3}}\right)\right\}^{-1 / 4} .
$$

Integrating from 0 to $T_{s}$, we have

$$
\begin{aligned}
\int_{0}^{T_{s}}\left\{\tilde{\mathcal{V}}\left(t_{s}\right)^{4 / 3} \ln \left(\frac{\tilde{\mathcal{V}}\left(t_{s}\right)}{h_{\text {avg }}^{3}}\right)\right\}^{-p / 4} d t_{s} & \geqslant 3^{-p / 4} h_{\text {avg }}^{-p} \int_{0}^{T_{s}} \tilde{\Phi}\left(t_{s}\right)^{p} d t_{s} \\
& \geqslant 3^{-p / 4} C_{2} \int_{0}^{T_{s}} t_{s}^{-p / 4} d t_{s}
\end{aligned}
$$

provided $T_{s} \geqslant C_{1} h_{\text {avg }}^{4} \tilde{S}(0)^{4}$. The last inequality in (3.42) follows from (3.31).

REMARK 3.8. For the average radius $\mathcal{R}_{\text {avg }}$, we expect an upper bound in the form of a power-logarithmic law

$$
\mathcal{R}_{\text {avg }}\left(\ln \mathcal{R}_{\text {avg }}\right)^{1 / 4} \leqslant c t^{1 / 4}
$$

which is consistent with (1.1). However, this is not trivial even if we have the inequalities (3.33). The difference between $\mathcal{R}_{\text {avg }}$ and $\mathcal{V}_{\text {avg }}^{1 / 3}$ is that the latter is always 
increasing while the former need not necessarily be monotone. The monotonicity is crucial since in the proof of Theorem 1.2 we used the assumption that $\mathcal{V}_{\text {avg }}^{1 / 3}>h_{\text {avg }}$, which is guaranteed to be true as long as it is satisfied in the initial configuration. It is not clear to us how to obtain a similar relation $\mathcal{R}_{\text {avg }}>h_{\text {avg }}$ for all time, even though heuristically it should be true.

REMARK 3.9. For the critical radius $\mathcal{R}_{*}$, we do not expect any power-logarithmic law. In fact since $\mathcal{R}_{*}$ is the harmonic mean of $\left\{R_{i}\right\}$, it always approaches 0 as some droplets shrink and disappear. This singularity generates many more difficulties than the failure of the logarithm.

\section{Self similarity and the structural time scale}

Experiments and numerical simulations indicate that the distribution of the sizes of droplets exhibits some self similarity (see, e.g., [13] and references therein). Mathematically, we do not know if the distribution always approaches a self similar solution. For the Ostwald ripening for phase transitions, it is known that distributions of droplets may not always approach a self similar solution [19].

Our mean field model provides a convenient way to study the self similarity of distributions of droplets, since the distribution $f(t, R)$ must satisfy the transport equation

$$
\begin{gathered}
\frac{\partial}{\partial t} f(t, R)+\frac{\partial}{\partial R}\left(-\frac{1}{R^{2}} \frac{1}{\ln \phi^{-1}}\left(\frac{1}{R}-P_{*}\right) f(t, R)\right)=0, \\
\phi=\frac{\int_{0}^{\infty} R^{2} f(t, R) d R}{\mathcal{L}^{2}}, \quad P_{*}=\frac{\int_{0}^{\infty} R^{-1} f(t, R) d R}{\int_{0}^{\infty} f(t, R) d R},
\end{gathered}
$$

with the requirements that $\int_{0}^{\infty} R^{2} f(t, R) d R \leqslant e^{-1} \mathcal{L}^{2} \quad$ (since we require $\phi \leqslant e^{-1}$ in Theorem 1.1) and that $\mathcal{V}_{\text {total }}=\int_{0}^{\infty} R^{3} f(t, R) d R$ is conserved.

The properties of the transport Equation (4.1)-(4.2) are beyond the scope of this paper. The rest of this section is about some properties of the structural time scale $t_{s}$.

As is discussed in Section 3, we already know that the structural time scale $t_{s}$ is not less than $t$, i.e., $t_{s} \geqslant t$. In addition, $t_{s}$ has some connection with the distribution of radii of droplets. In fact we have the following

Proposition 4.1. If the distribution of radii is self similar, then in the dilute limit when the fraction $\phi \rightarrow 0$, we have

$$
\frac{d t_{s}}{d t}=1+o(1)
$$

Hence

$$
t_{s}=(1+o(1)) t \quad \text { as } \phi \rightarrow 0 .
$$

Proof. If $f(t, R)$ is a self-similar solution of the mean field model, since the total volume, which is the third moment, is conserved, there must be some function $F(\cdot)$ and some positive constant $a$ and some positive function $b(t)$ such that

$$
f(t, R)=a b(t)^{-4} F(R / b(t))
$$


By the definitions of $\phi(t)$ and $\sigma(t)$ (Equations (1.3) and (1.12)), we have

$$
\begin{aligned}
\phi(t) \sigma(t) & =\frac{\left(\int_{0}^{\infty} R^{2} f(t, R) d R\right)\left(\int_{0}^{\infty} R^{4} f(t, R) d R\right)}{\left(\int_{0}^{\infty} R^{3} f(t, R) d R\right)^{2}} \\
& =\frac{\left(\int_{0}^{\infty} R^{2} F(R / b(t)) d R\right)\left(\int_{0}^{\infty} R^{4} F(R / b(t)) d R\right)}{\left(\int_{0}^{\infty} R^{3} F(R / b(t)) d R\right)^{2}} \\
& =\frac{\left(\int_{0}^{\infty} u^{2} F(u) d u\right)\left(\int_{0}^{\infty} u^{4} F(u) d u\right)}{\left(\int_{0}^{\infty} u^{3} F(u) d u\right)^{2}} \\
& =: \beta .
\end{aligned}
$$

Here $\beta \geqslant 1$ is a positive constant depending only on $F$. Hence $\sigma(t)=\beta \phi(t)^{-1}$ and by the definition of $t_{s}$ (Equation (1.14)),

$$
\begin{aligned}
\frac{d t_{s}}{d t} & =\left(\frac{\ln \sigma(t)}{\ln \phi(t)^{-1}}\right)^{1 / 2}=\left(\frac{\ln \phi(t)^{-1}+\ln \beta}{\ln \phi(t)^{-1}}\right)^{1 / 2}=\left(1+\frac{\ln \beta}{\ln \phi(t)^{-1}}\right)^{1 / 2} \\
& =1+o(1) \quad \text { when } \phi=o(1) .
\end{aligned}
$$

Consequently $t_{s}=(1+o(1)) t$ as $\phi \rightarrow 0$.

REMARK 4.2. If the distribution is not self similar,

$$
\phi(t) \sigma(t)=\frac{\left(\int_{0}^{\infty} R^{2} f(t, R) d R\right)\left(\int_{0}^{\infty} R^{4} f(t, R) d R\right)}{\left(\int_{0}^{\infty} R^{3} f(t, R) d R\right)^{2}}=\beta(t)
$$

and we still have

$$
\frac{d t_{s}}{d t}=\left(1+\frac{\ln \beta(t)}{\ln \phi(t)^{-1}}\right)^{1 / 2}=1+o(1) \quad \text { when } \phi=o(1)
$$

as long as $\beta(t)$ is not very big. We do not know if $\beta(t)$ can blow up in some special situations but heuristically it does not seem to happen, as experiments and numerical simulations of thin film coarsening both indicate that the distributions approach some self similar solutions.

\section{Conclusions and discussions}

We proposed a mean field model for the Ostwald ripening of thin films and studied its coarsening rate. We defined a structural time scale which is heuristically similar to $t$ and captured the logarithmic factor in the spatio-temporal relation.

In our model the migration and collision of droplets are ignored. We expect to generalize our model to incorporate the migration and collisions, which can be significant for the coarsening in thin films.

On the other hand, mean field models simplify the PDE models for thin films. The PDEs naturally deal with topological changes in thin films without differentiating the ripening and collision mechanisms. It is considerably much more complicated since it is a challenge to retrieve statistical information in a PDE setting. Nevertheless, we are studying the possibility of a PDE version of the results in this paper. 


\section{REFERENCES}

[1] S. Conti, B. Niethammer and F. Otto, Coarsening rates in off-critical mixtures, SIAM J. Math. Anal., 37(6), 1732-1741, 2006.

[2] S. Dai, On a mean field model for $1 D$ thin film droplet coarsening, Nonlinearity, 23, 325-340, 2010.

[3] S. Dai, On the shortening rate of collections of plane convex curves by the area-preserving mean curvature flow, SIAM J. Math. Anal., 42(1), 323-333, 2010.

[4] S. Dai, B. Niethammer and R.L. Pego, Crossover in coarsening rates for the monopole approximation of the mullins-sekerka model with kinetic drag, Proc. Royal Soc. Edinburgh, Vol 140, Issue 03 (2010), 553-571.

[5] S. Dai and R.L. Pego, Universal bounds on coarsening rates for means field models of phase transitions, SIAM J. Math. Anal., 37(2), 347-371, 2005.

[6] S. Dai and R.L. Pego, An upper bound on the coarsening rate for mushy zones in a phase field model, Interfaces and Free Boundaries, 7, 187-197, 2005.

[7] S. Esedoglu and J.B. Greer, Upper bounds on the coarsening rate of discrete, ill-posed nonlinear diffusion equations, Commun. Pure. Appl. Math., 62(1), 57-81, 2009.

[8] S. Esedoglu and D. Slepčev, Refined upper bounds on the coarsening rate of discrete, ill-posed diffusion equations, Nonlinearity, 21(12), 2759-2776, 2008.

[9] K.B. Glasner, Ostwald ripening in thin film equations, SIAM J. Appl. Math., 69(2), 473-493, 2008.

[10] K.B. Glasner and T.P. Witelski, Coarsening dynamics of dewetting films, Phys. Rev. E, 67(1), 016302, 2003.

[11] Karl Glasner, Felix Otto, Tobias Rump and Dejan Slepčev, Ostwald ripening of droplets: the role of migration, Euro. J. Appl. Math., 20, 1-67, 2009.

[12] K.B. Glasner and T.P. Witelski, Collision versus collapse of droplets in coarsening of dewetting thinfilms, Physica D: Nonlinear Phenomena, 209(1-4), 80-104, 2005.

[13] Michael B. Gratton and Thomas P. Witelski, Transient and self-similar dynamics in thin film coarsening, Physica D: Nonlinear Phenomena, 238(23-24), 2380-2394, 2009.

[14] R.V. Kohn and F. Otto, Upper bounds on coarsening rates, Commun. Math. Phys., 229, 375$395,2002$.

[15] R.V. Kohn and X. Yan, Upper bounds on the coarsening rate for an epitaxial growth model, Commun. Pure App. Math., 56(11), 1549-1564, 2003.

[16] R.V. Kohn and X. Yan, Coarsening rates for models of multicomponent phase separation, Interfaces and Free Boundaries, 6, 135-149, 2004.

[17] B. Niethammer and F. Otto, Domain coarsening in thin films, Commun. Pure. Appl. Math., 54(3), 361-384, 2001.

[18] B. Niethammer and F. Otto, Ostwald ripening: the screening length revisited, Calc. Var. and PDE, 13(1), 33-68, 2001.

[19] B. Niethammer and R.L. Pego, Non-self-similar behavior in the LSW theory of Ostwald ripening, J. Stat. Phys., 95, 867-902, 1999.

[20] A. Oron, S.H. Davis and S.G. Bankoff, Long-scale evolution of thin liquid film, Rev. Mod. Phys., 69, 931-980, 1997.

[21] Felix Otto, Tobias Rump and Dejan Slepčev, Coarsening rates for a droplet model: rigorous upper bounds, SIAM J. Math. Anal., 38(2), 503-529 (electronic), 2006.

[22] P.W. Voorhees, The theory of Ostwald ripening, J. Stat. Phys., 38(1/2), 231-252, 1985. 